\title{
Crystal structure of the INTS3/INTS6 complex reveals the functional importance of INTS3 dimerization in DSB repair
}

\author{
Yu Jia', Zixiu Cheng ${ }^{1}$, Sakshibeedu R. Bharath², Qiangzu Sun ${ }^{1}$, Nannan Su', Jun Huang $\mathbb{D}^{1}$ and Haiwei Song (1) ${ }^{1,2,3}$
}

\begin{abstract}
SOSS1 is a single-stranded DNA (ssDNA)-binding protein complex that plays a critical role in double-strand DNA break (DSB) repair. SOSS1 consists of three subunits: INTS3, SOSSC, and hSSB1, with INTS3 serving as a scaffold to stabilize this complex. Moreover, the integrator complex subunit 6 (INTS6) participates in the DNA damage response through direct binding to INTS3, but how INTS3 interacts with INTS6, thereby impacting DSB repair, is not clear. Here, we determined the crystal structure of the C-terminus of INTS3 (INTS3C) in complex with the C-terminus of INTS6 (INTS6C) at a resolution of $2.4 \AA$. Structural analysis revealed that two INTS3C subunits dimerize and interact with INTS6C via conserved residues. Subsequent biochemical analyses confirmed that INTS3C forms a stable dimer and INTS3 dimerization is important for recognizing the longer ssDNA. Perturbation of INTS3C dimerization and disruption of the INTS3c/INTS6c interaction impair the DSB repair process. Altogether, these results unravel the underappreciated role of INTS3 dimerization and the molecular basis of INTS3/INTS6 interaction in DSB repair.
\end{abstract}

\section{Introduction}

DNA double-strand breaks (DSBs) arise when the integrity of genomic DNA is challenged by a variety of endogenous and exogenous DNA-damaging agents such as replication fork collapse, oxidative stress, and ionizing radiation $(\mathrm{IR})^{1}$. DSBs are highly cytotoxic lesions that disrupt the continuity of a chromosome ${ }^{2}$. Defective DSB repair leads to genome instability and is associated with developmental disorders, premature aging, and tumorigenesis ${ }^{3,4}$.

In eukaryotes, two main pathways, homologous recombination (HR) and non-homologous end-joining (NHEJ), have evolved to repair $\mathrm{DSBs}^{5-7}$. As a more accurate

\footnotetext{
Correspondence: Jun Huang (jhuang@zju.edu.cn) or

Haiwei Song (haiwei@imcb.a-star.edu.sg)

${ }^{1}$ MOE Laboratory of Biosystems Homeostasis and Protection and Innovation Center for Cell Signaling Network, Life Sciences Institute, Zhejiang University, Hangzhou, Zhejiang, China

${ }^{2}$ Institute of Molecular and Cell Biology, 61 Biopolis Drive, Singapore City, Singapore

Full list of author information is available at the end of the article

These authors contributed equally: Yu Jia, Zixiu Cheng, Sakshibeedu R. Bharath
}

mechanism, HR requires a sister chromatid as the template to ensure genome integrity, but only occurs in the $S$ and G2 phases of the cell cycle ${ }^{8}$. One of the initial steps in the process of $\mathrm{HR}$ is the resection of DSBs to generate a $3^{\prime}$ single-stranded DNA (ssDNA) overhang, which is essential for Rad51-mediated strand exchange ${ }^{9-11}$. Following the resection of DSBs, the replication protein A (RPA) complex immediately binds to these ssDNA overhangs, thereby not only protecting these ssDNA regions from nucleases but also facilitating Rad51 filament formation ${ }^{12}$. In addition to RPA, human SSB1 and SSB2 (hSSB1, hSSB2) have been shown to be involved in DNA repair mechanisms in eukaryotic cells ${ }^{13}$. These SSBs possess an oligonucleotide/oligosaccharide-binding (OB) fold, which facilitates protein-nucleic acid and protein-protein interactions. Following DNA damage, hSSB1 is stabilized by ATM-dependent phosphorylation and localizes to the sites of damaged DNA as discrete foci ${ }^{13-15}$.

We and others have shown that hSSB1 and hSSB2 form an independent trimeric complex referred to as SOSS1 or SOSS2, with two other proteins, integrator complex

\section{(c) The Author(s) 2021}

(c) (i) Open Access This article is licensed under a Creative Commons Attribution 4.0 International License, which permits use, sharing, adaptation, distribution and reproduction cc) in any medium or format, as long as you give appropriate credit to the original author(s) and the source, provide a link to the Creative Commons license, and indicate if changes were made. The images or other third party material in this article are included in the article's Creative Commons license, unless indicated otherwise in a credit line to the material. If material is not included in the article's Creative Commons license and your intended use is not permitted by statutory regulation or exceeds the permitted use, you will need to obtain permission directly from the copyright holder. To view a copy of this license, visit http://creativecommons.org/licenses/by/4.0/. 
subunit 3 (INTS3) and SOSSC ${ }^{16-18}$. We reconstituted a SOSS1 subcomplex containing the N-terminal half of INTS3 (INTS3 ${ }_{\mathrm{N}}$ ) and full-length hSSB1, and a trimeric complex composed of INTS3 ${ }_{\mathrm{N}} / \mathrm{hSSB} 1$ and full-length SOSSC. We determined the crystal structures of the trimeric complex (INTS3 $3_{\mathrm{N}} / \mathrm{hSSB} 1 / \mathrm{SOSSC}$ ) alone and in complex with a 35-nt ssDNA, as well as the structure of the dimeric complex (INTS3 ${ }_{N} / \mathrm{hSSB} 1$ ) bound to a 12 -nt ssDNA $^{19}$. These structures combined with functional analysis confirmed that INTS3 ${ }_{\mathrm{N}}$ acts as a scaffold to bridge the interaction between hSSB1 and SOSSC, and showed that hSSB1 binds to INTS3 ${ }_{\mathrm{N}}$ and ssDNA through two distinct surfaces ${ }^{19}$. Recent biochemical characterization of INTS3 showed that the C-terminus of INTS3 (INTS3c) is required for nucleic acid binding ${ }^{20}$. However, how INTS3 contributes to ssDNA binding and the mechanism by which SOSS1 recognizes longer ssDNA remain elusive. Stringent affinity purification coupled with mass spectrometry experiments suggested yet another protein, named integrator complex subunit 6 (INTS6), to be associated with the $\mathrm{SOSS}^{21}$. In response to DSBs, INTS6 is recruited to DSBs by directly interacting with INTS3c. Depletion of INTS6 and its paralog DDX26b abolished RAD51 foci formation, suggesting that the recruitment of INTS6 to DSBs along with INTS3 and hSSB1 regulates the accumulation of RAD51 and the subsequent HR repair ${ }^{21}$.

To clarify the molecular mechanism underlying the assembly of the INTS3/INTS6 complex in DSB repair, we crystallized and determined the crystal structure of the INTS3c (residues 560-905, 915-995)/INTS6c (residues 800-887) complex. The structure showed that INTS3c forms a dimer and both subunits mediate its interaction with INTS6c. Biochemical assays further confirmed that INTS3 functions as a dimer. Structurebased mutagenesis indicated that mutations that disrupt the INTS3 dimer or the INTS3c/INTS6c interactions impaired RAD51 foci formation and sensitized cells to IR. Dimerization of INTS3 is also required for the recognition of longer ssDNA. Overall, these findings highlight the important role of the INTS3 dimer and the molecular basis of the INTS3/INTS6 interaction in DSB repair.

\section{Results}

\section{Overall structure of the INTS3c/INTS6c complex}

The purified C-terminal half of INTS3 (560-995) was subjected to limited proteolysis followed by $\mathrm{N}$-terminal sequencing to identify two stable fragments of INTS3, residues 560-905 and residues 915-995 (Fig. 1a). These fragments were co-expressed and purified along with Cterminal residues $800-887$ of INTS6 (Fig. 1a) to yield a homogenous, stable, and crystallizable INTS3c/INTS6c complex (Supplementary Fig. S1). Analytical size-exclusion chromatography suggested that the INTS3c/INTS6c complex was eluted as a dimer (Supplementary Fig. S2). The crystal structure of selenomethionine-substituted INTS3c in complex with INTS6c was determined using the single-wavelength anomalous dispersion (SAD) method. The structure of the INTS3c/INTS6c complex reveals an all $\alpha$-helical assembly resembling the shape of a "butterfly", with the INTS3c dimer forming the wings and one INTS6c subunit near the two-fold axis of the dimer interface making the body of the butterfly (Fig. 1b). The molar ratio between INTS3c and INTS6c is 2:1. INTS3c adopts a right-handed super-helical structure with 20 helices, in which each helix is paired with its neighboring helix to form an antiparallel helix pair (Fig. 1b). INTS6c contains four $\alpha$-helices (Fig. 1a) and interacts with INTS3c via two loops between $\alpha 1-\alpha 2$ and $\alpha 3-\alpha 4$.

\section{The INTS3c dimer interface}

Two INTS3c protomers form a symmetrical dimer (Fig. 1b) wherein two protomers are related by a noncrystallographic two-fold axis. Analysis of the dimer interface using Protein Interfaces, Surfaces, and Assemblies webserver (PISA) ${ }^{22}$ revealed a buried surface area of $\sim 3400.0 \AA^{2}$. Each protomer contributes 46 residues to the dimer interface, forming a vast network of hydrophobic, van der Waals, and hydrogen bond interactions with a free energy of dissociation, $\Delta G^{\text {diss }}$, of $9.7 \mathrm{kcal} / \mathrm{mol}$. The interface mainly consists of residues belonging to four helical segments (Fig. 1c), hA (Asp769-Leu785), hB (Glu804-Ile820), hC (Lys833-Lys852), and hD (Pro868-His884). The bulk of the hydrophobic core consists of Leu773, Phe806, Tyr809, and Leu846 from both chains of the dimer (Fig. 1c). These interactions are further strengthened by additional hydrophobic interactions, 22 hydrogen bonds, and numerous van der Waals interactions, e.g., Met782 of hA contacts Gln871 of hD, and Gln774 of hA contacts Leu845 of hC. The tight INTS3c dimer and the conserved residues in the interface (Supplementary Fig. S3a) suggest that the dimer likely corresponds to a physiologically relevant form of INTS3.

\section{The INTS3/INTS6 interface}

The interactions of INTS6c with chains A and B of INTS3c account for a total buried surface area of 706.8 and $503.5 \AA^{2}$, respectively. The interface is rather small with only the loops between the helical segments of INTS6c interacting with the INTS3c dimer. As shown in Fig. 2a, the core of the interface is hydrophobic in nature with Pro823 and Phe858 of INTS6c juxtaposed against Phe806 from the two subunits of INTS3c. The interaction is further augmented by salt bridges between Lys859 of INTS6c and Glu839 of INTS3c, Arg825 of INTS6c and Glu804 and Glu807 of INTS3c, and Arg857 of INTS6c and Asp769 of INTS3c. Sequence alignment of INTS6c from human, mouse, 

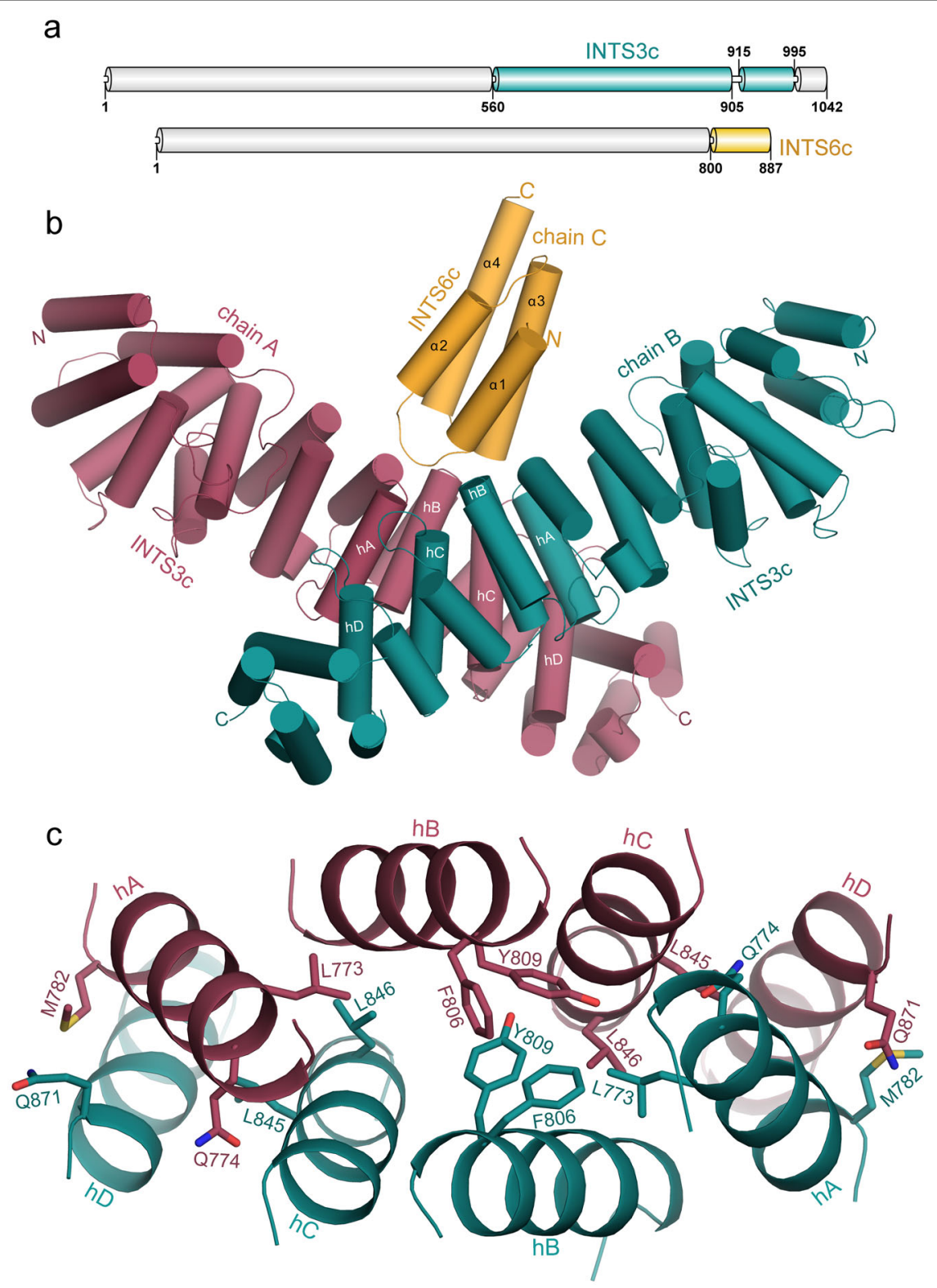

Fig. 1 Structure of the INTS3c/INTS6c complex. a Schematic representation of human INTS3 and INTS6. b Crystal structure of the INTS3c/INTS6c complex. c The dimer interface of INTS3c. The central core of the dimeric interface of INTS3c is made up of helical segments: hA, hB, hC, and hD. For simplicity of viewing, only few interactions involving Leu773, Gln774, Met782, Phe806, Tyr809, Leu845, Leu846, Gln871, and their dyad symmetry mates are shown.

zebrafish, and Drosophila species (Supplementary Fig. S3b) highlighted the conserved nature of the residues involved in the interaction with INTS3c.

\section{Mutational analysis}

To identify the residues important for INTS3c/ INTS6c interaction, we prepared a number of sitespecific mutations in either INTS3c or INTS6c and examined the binding of these mutants to their respective wild-type binding partner using GST-pull down assays. Substitution of amino acids Phe858 and Lys859 to Ala (F858A/K859A) in INTS6c abolished its binding to INTS3c (Fig. 2b). Conversely, the substitution of amino acids Phe806 and Glu839 to Ala (F806A/ E839A) or substitution of amino acids Val767 and Asp769 to Ala (V767A/D769A) in INTS3c substantially diminished its interaction with INTS6c (Fig. 2c).

We further introduced these mutations into full-length INTS3 and INTS6 and tested their interaction by coimmunoprecipitation assays. As shown in Fig. 2d, the 


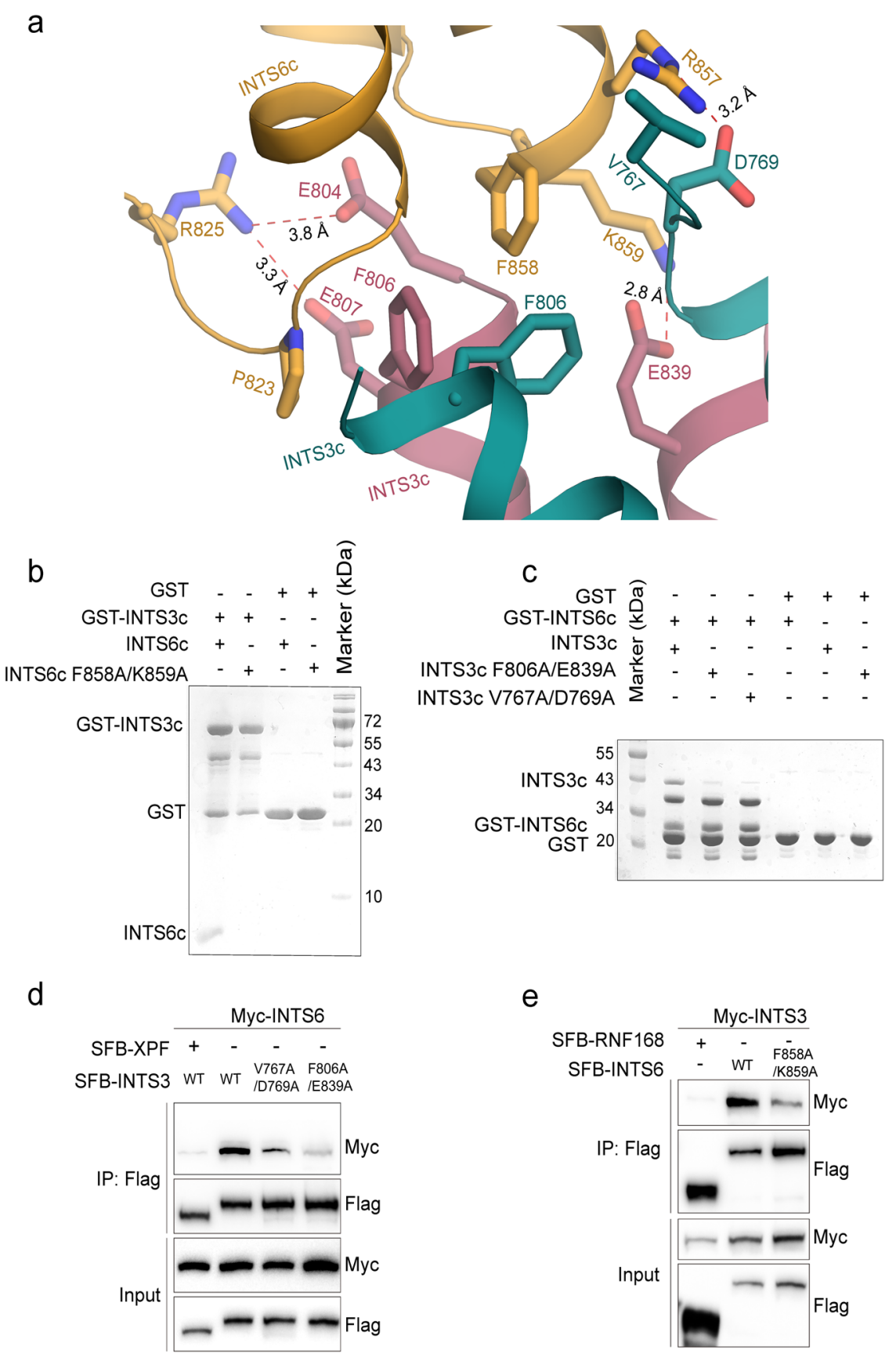

Fig. 2 Interface between INTS3c and INTS6c. a The INTS3c/NTS6c interface is composed of central hydrophobic residues, Pro823, Phe858 from INTS6c, and Phe806 from INTS3c. These hydrophobic interactions are further strengthened by salt bridges and hydrogen bonds. $\mathbf{b}$ GST-pull down assays show that the F858A/K839A mutant of INTS6c did not interact with INTS3c. c Results of GST-pull down assays show that the V767AVD769A and F806AVE839A mutants of INTS3C did not interact with INTS6C. d The V767AVD769A and F806A/E839A mutants of full-length INTS3 show weak interaction with full-length INTS6 in HEK293T cells that were transfected with plasmids encoding Myc-tagged INTS6 and SFB-tagged wild-type INTS3 (WT) or its mutants. SFB-tagged XPF was used as a negative control. Cell lysates were immunoprecipitated with anti-Flag antibody and western blot analysis was performed with anti-Flag and antiMyc antibodies. e The F858A/K859A mutant of full-length INTS6 shows weak interaction with full-length INTS3. Co-immunoprecipitation experiments were performed similarly to those described in d. SFB-tagged RNF168 was used as a negative control.

V767A/D769A or F806A/E839A mutant of INTS3 showed dramatically weakened interaction with INTS6. Similarly, the F858A/K859A mutant of INTS6 also exhibited reduced binding to INTS3 (Fig. 2e). Altogether, these results indicate that these residues are indeed important for the physical interaction between INTS3 and INTS6. 


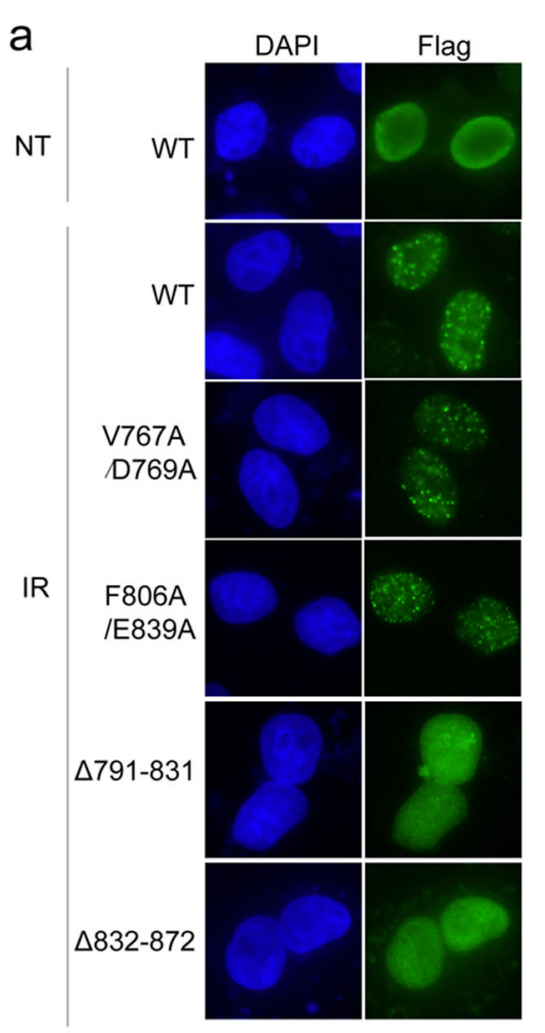

b

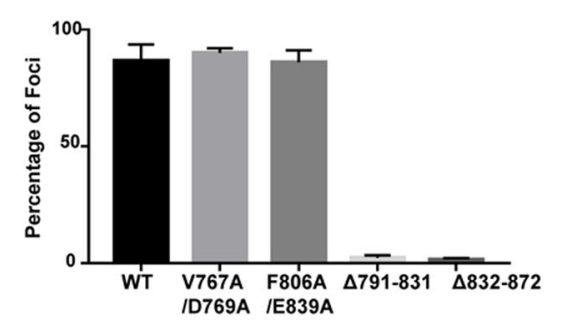

C

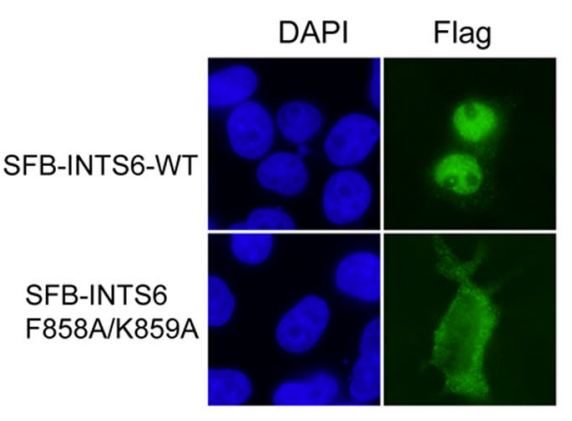

Fig. 3 The effects of INTS3 dimerization and its interaction with INTS6 on INTS3/INTS6 recruitment to DSB sites. a Cells transfected with SFBtagged wild-type INTS3 or its mutants were treated with IR (10 Gy) for $6 \mathrm{~h}$ or left untreated. Cells were then fixed and processed for immunofluorescence with an anti-Flag antibody. $\mathbf{b}$ The quantification of foci-positive cells was performed by counting a total of 300 cells per sample. c Cells transfected with SFB-tagged wild-type INTS6 or the F858A/K859A mutant were fixed and processed for immunofluorescence with anti-Flag antibody.

Next, we examined whether the INTS3/INTS6 interaction is important for their recruitment to DNA damage sites. As shown in Fig. $3 \mathrm{a}$ and $\mathrm{b}$, similar to wild-type INTS3, both the V767A/D769A and F806A/E839A mutants could still relocate to DSB sites following IR treatment. In contrast, the $\mathrm{F} 858 \mathrm{~A} / \mathrm{K} 859 \mathrm{~A}$ mutant of INTS6 showed a diffuse cytoplasmic localization, suggesting that forming a complex with INTS3 is essential for INTS6 nuclear localization (Fig. 3c). These results are consistent with the previous finding that INTS3 recruits INTS6 to DSB sites in response to $\mathrm{IR}^{21}$. We further tested whether INTS3 damage recruitment is dependent on its dimerization. To this end, we generated two deletion mutants rather than the site-directed mutants due to the extensive interface of the INTS3 dimer. Specifically, we separately deleted residues 791-831 and residues 832-872 (designated as INTS3 ${ }^{\Delta 791-831}$ and INTS3 ${ }^{\Delta 832-872}$ ), which contain helices $\mathrm{hB}$ and $\mathrm{hC}$ critical for INTS3 dimerization (Fig. 1c). As shown in Fig. 3a and b, both the deletion mutants of INTS3 failed to form IR-induced foci, indicating that dimerization is necessary for INTS3 recruitment to DSB sites.
To investigate the biological significance of INTS3 dimerization and its interaction with INTS6, we utilized a doxycycline-inducible expression system to express the siRNA-resistant wild-type INTS3, INTS6, or the mutants defective in INTS3 dimerization or INTS3/INTS6 interaction in INTS3- or INTS6-depleted cells. As shown in Fig. 4a and b, neither the V767A/D769A and F806A/ E839A mutants nor the INTS3 ${ }^{\Delta 791-831}$ and INTS3 ${ }^{\Delta 832-872}$ mutants were able to restore RAD51 foci formation in INTS3-depleted cells. Likewise, RAD51 foci formation in INTS6-depleted cells could be restored by wild-type INTS6, but not the F858A/K859A mutant (Fig. 4c, d). More importantly, perturbation of INTS3 dimerization or disruption of the INTS3-INTS6 complex formation rendered cells more sensitive to IR (Fig. 4e, f). Taken together, these results suggest that both INTS3 dimerization and the INTS3/INTS6 complex formation are important for DSB repair.

\section{Recognition of ssDNA}

Our previous structural studies of the SOSS1 complex $^{19}$ did not include the C-terminal domain of INTS3, 


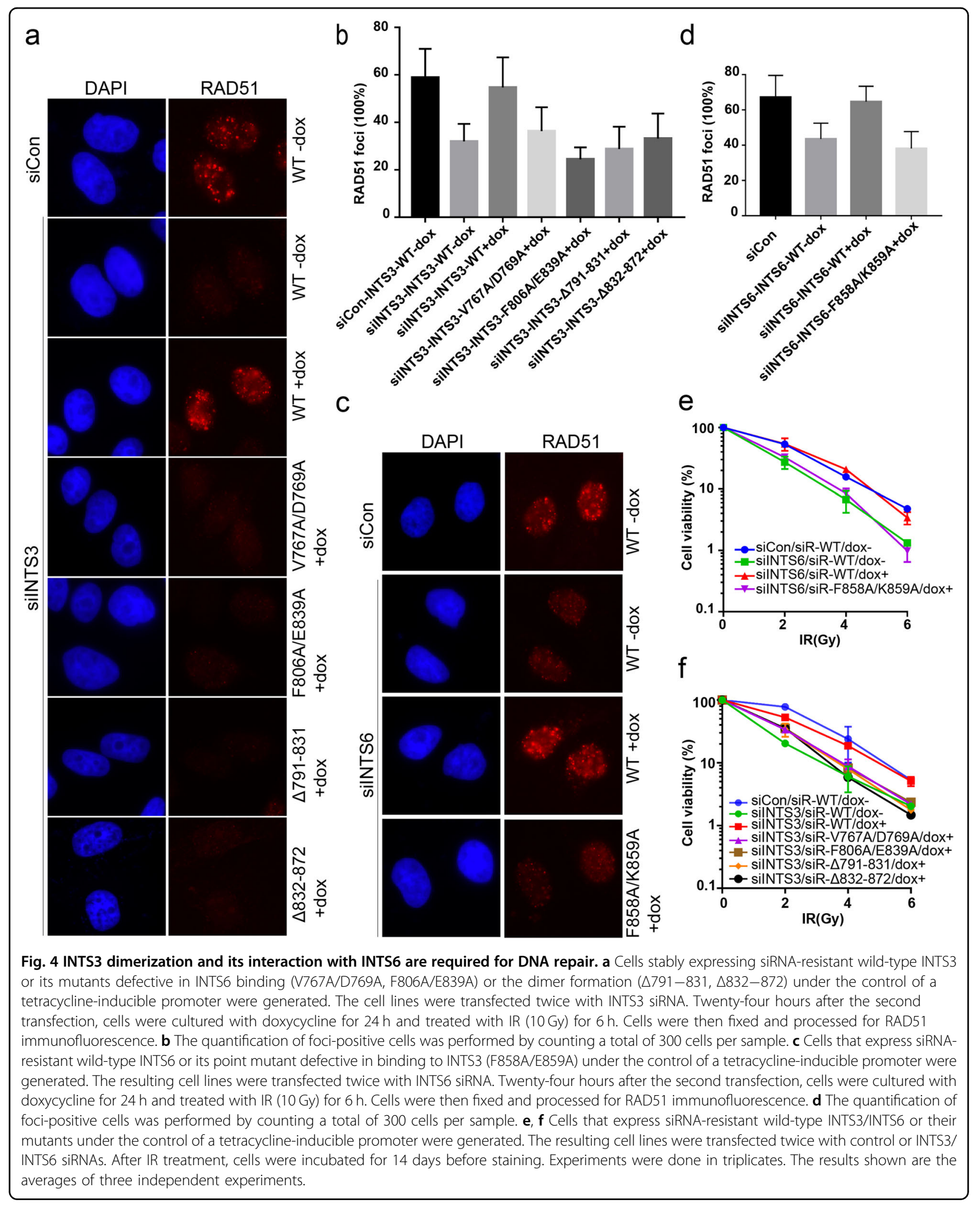


resulting in a poor understanding of long ssDNA recognition. Moreover, the C-terminal of INTS3 has been reported to be required for ssDNA binding ${ }^{20}$. To explore the function of INTS3 dimer in recognition of longer ssDNA, we created a series of deletion mutants of INTS3 to test if its dimerization could improve longer ssDNA binding by electrophoretic mobility shift assay (EMSA).

Wild-type hSSB1, INTS3(1-995)-hSSB1, and INTS3(1500)-hSSB1 were co-expressed and purified (Supplementary Fig. S4a) and subjected to analytical size-exclusion chromatography (SEC) analysis. INTS3(1-995)-hSSB1 contains residues of 1-995 (deletion of the C-terminal unstructured region; residues 996-1042) and a full-length hSSB1 while INTS3(1-500)-hSSB1 consists of just INTS $3_{\mathrm{N}}$ and a full-length hSSB1 and has been shown to be in a heterodimeric form in solution previously ${ }^{19}$. As expected, INTS3(1-995)-hSSB1 forms a dimer of heterodimer while INTS3(1-500)/hSSB1 is a heterodimer in solution (Supplementary Fig. S2). To examine whether dimerization affects the binding of longer ssDNA, we used SPR assays to check the binding of dT48 to hSSB1, INTS3(1-500)hSSB1, and INTS3(1-995)-hSSB1. The results showed that hSSB1 and INTS3(1-500)/hSSB1 bind to dT48 with comparable affinities while INTS3(1-995)-hSSB1 and fulllength INTS3/hSSB1 bind to dT48 with about 4-5 times higher affinities than that of hSSB1 alone or INTS3(1500)/hSSB1 (Supplementary Fig. S5). EMSA assays showed that INTS3(1-995)-hSSB1, INTS3(1-500)-hSSB1, and hSSB1 bind to dT12 with comparable affinities (Supplementary Fig. S4c). Moreover, the presence of INTS6c had no effect on the binding of INTS3(1-995)-hSSB1 to dT48 (Fig. 5a, b), suggesting that INTS6c is not required for ssDNA binding. To further clarify the role of INTS3 dimerization in the binding of longer ssDNA, we coexpressed INTS3 ${ }^{\Delta 791-831}$ and INTS3 ${ }^{\Delta 832-872}$ in the context of a truncated INTS3 (residues 1-995) with hSSB1 (termed as INTS3 $(1-995)^{\Delta 791-831}$-hSSB1 and INTS3 $(1-995)^{\Delta 832-872}$-hSSB1 respectively). Purified INTS3

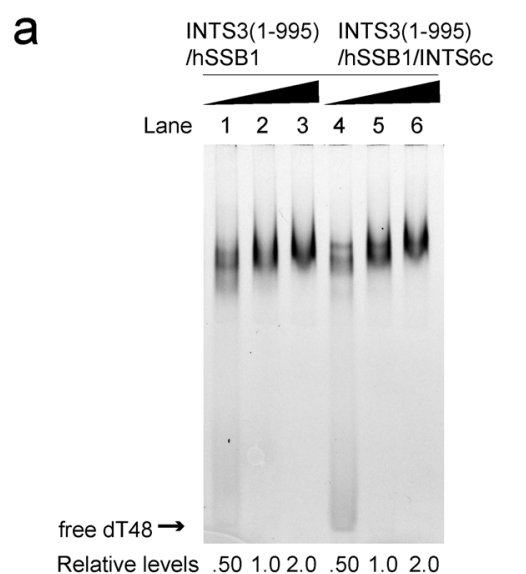

b
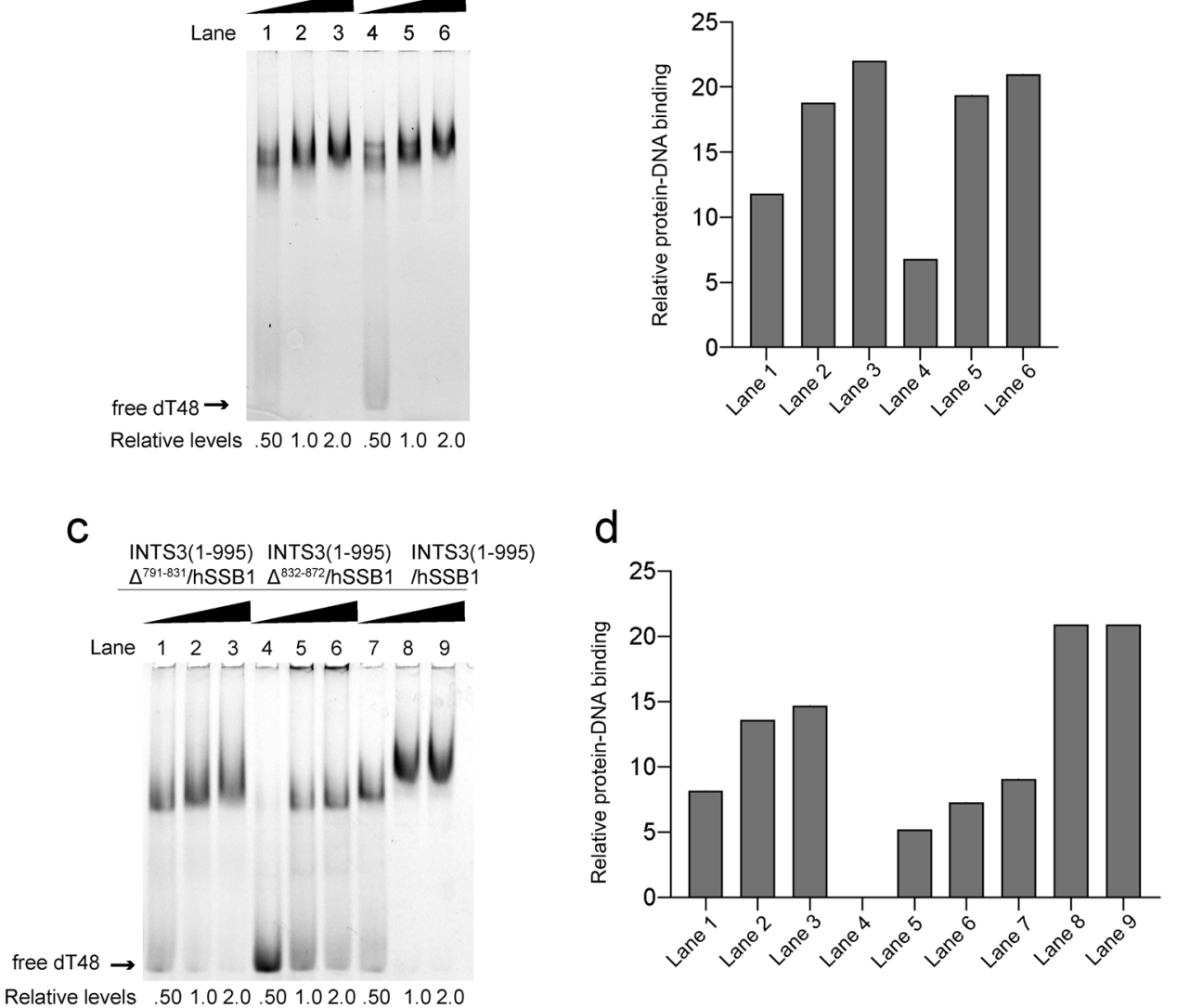

Fig. 5 Interaction of hSSB1 and other INTS3/hSSB1 complexes with ssDNA. a Interaction of dT48 with INTS3 (1-995)/hSSB1 and INTS3 (1-995)/ hSSB1/INTS6C as examined by electrophoretic mobility assay (EMSA). b Quantification of relative protein-DNA binding in a. c Interaction of dT48 with INTS3 (1-995) ${ }^{\Delta 791-831}$ /hSSB1, INTS3 (1-995) ${ }^{\triangle 832-872} / \mathrm{hSSB1}$ and INTS3 (1-995)/hSSB1 as examined by electrophoretic mobility assay (EMSA). d Quantification of relative protein-DNA binding (c). 
$(1-995)^{\Delta 791-831}-\mathrm{hSSB} 1$ and INTS3 $(1-995)^{\Delta 832-872}$ hSSB1 showed similar proteolytic patterns to that of wildtype INTS3 (1-995)-hSSB1, suggesting that the deletions in the INTS3 dimer interface did not affect the protein folding so much (Supplementary Fig. S4b). EMSA experiments showed that both INTS3 (1-995) ${ }^{\Delta 791-831}$ hSSB1 and INTS3 (1-995) ${ }^{\Delta 832-872}$-hSSB1 exhibited reduced binding to dT48 compared to INTS3(1-995)hSSB1 (Fig. 5c, d). Altogether, these results strongly suggest that the dimerization of INTS3 is required for the binding of the longer ssDNA but has no effect on the short ssDNA binding.

\section{Mechanistic insights into longer ssDNA recognition by SOSS1 complex}

A striking feature of the structure of the INTS3c/ INTS6c complex is the formation of an X-shaped INTS3 dimer. Importantly, we demonstrated the functional importance of INTS3 dimerization in DSB repair as well as in recognizing the longer ssDNA. Our previous crystal structure of $\mathrm{INTS}_{\mathrm{N}} / \mathrm{SSB} 1 / \mathrm{SOSSC}$ showed that the recognition of ssDNA with a length up to nine nucleotides is solely mediated by a single OB domain of hSSB $1^{19}$. But, how the full-length SOSS1 complex recognizes the longer ssDNA up to 35 nucleotides remains unclear. hSSB1 is a monomer under reducing conditions ${ }^{13,23}$. However, oxidation stress can promote hSSB1 to form a tetramer, involved in oxidative DNA damage repair and is similar to other tetrameric $\mathrm{SSBs}^{24,25}$. The tetrameric SSBs from $E$. coli (Ec-SSB) and P. falciparum (Pf-SSB) consist of two distinct dimer interfaces, an extensive interface formed by subunits I and II (or subunits III and IV) with a buried surface area of $1200 \AA^{2}$ and a significantly smaller interface formed by subunits II and III (or subunits I and IV) with a buried surface area of $600 \AA^{226,27}$. The I-II SSB dimer binds to a 35-nt ssDNA in $E c$-SSB and $P f-S S B^{26,27}$, suggesting that this SSB dimer is functionally important. Further support for this notion comes from the structure of $D$. radiodurans SSB, wherein a single polypeptide chain forms two OB domains-analogous to the I-II dimer in $E c$-SSB and Pf-SSB ${ }^{28}$.

We speculated that in the full-length SOSS1 complex, INTS3 dimerization would force hSSB1 to assemble into a dimer with its interface similar to the I-II interface in $E c$ SSB and Pf-SSB. To explore the mechanism governing the longer ssDNA recognition by the SOSS1 complex, we superimposed the hSSB1 subunit of INTS $3_{\mathrm{N}} / \mathrm{SSB} 1 /$ SOSSC onto each SSB monomer in the Pf-SSB tetramer as the polarity of ssDNA in the structure of $E c$-SSB is opposite to those in all other known structures of tetrameric SSBs. The results revealed that the superposition of hSSB1 subunit of INTS $3_{N} /$ SSB1/SOSSC onto subunits I and II or subunits III and IV leads to a plausible model, whereas other superpositions resulted in serious steric clashes. We then manually aligned the dyad axis of the hSSB1 dimer with that of the INTS3c dimer to generate the final INTS3/hSSB1/SOSSC/INTS6c model (Fig. 6). In support of the validity of this model, the ssDNA of $\sim 35$-nt modeled to hSSB1 contacts the residues of INTS $3_{\mathrm{N}}$ with the same polarity, which were previously observed in the structure of INTS $3_{\mathrm{N}} / \mathrm{SSB} 1 / \mathrm{SOSSC}^{19}$. Such a model could explain how SOSS1 binds to the longer ssDNA and at the same time allow hSSB1 to interact with the MRN complex via its $C$-terminal domain ${ }^{14,15}$. However, this model is not compatible with the disulfide-mediated hSSB1 tetramer assembled under oxidized conditions ${ }^{25}$. In line with this notion, tetramerization of hSSB1 has been shown to be dispensable for DSB repair ${ }^{24}$.

\section{Discussion}

Our previous study showed that INTS3 is a scaffold protein with its $\mathrm{N}$-terminal half-mediating the interaction with $\mathrm{hSSB} 1^{19}$. In this study, we showed that INTS3 uses its C-terminal region to mediate its interaction with INTS6. Consistent with the previous observations that the INTS3/INTS6 interaction is required for the recruitment of INTS6 to the DNA damage sites in response to $\mathrm{IR}^{21}$, our structure-based functional assays showed that the disruption of the INTS3c/INTS6c interaction inhibits the DSB repair process. Both INTS3 and INTS6 are the components in the Integrator complex participating in $3^{\prime}$ non-coding RNA processing ${ }^{29}$. Our studies showed that INTS6 interacts with INTS3 as a component of the integrator complex involved in DSB repair. In the transcription process, R-loop formed from RNA/DNA hybrids may lead to a pause in transcription and/or termination, resulting in genome instability ${ }^{30}$. The R-loops also accumulate at the DSB site ${ }^{31}$. INTS6 contains a DEAD (Asp -Glu-Ala-Asp) motif usually found in RNA/DNA helicases. A Von Willebrand factor type A domain is located at the N-terminal region of INTS6, which may also serve as a platform for protein-protein interaction ${ }^{32}$. This indicates that INTS3/INTS6/hSSB1 complex may play a role in binding and unwinding R-loop by recruiting other integrator members. Recently, INTS7 was found to interact with INTS3/hSSB1 as a whole complex in response to UV damage, and its depletion results in cell cycle arrest bypass and mitomycin $\mathrm{C}$ sensitivity ${ }^{33}$. Further studies are required to clarify the role of the INTS3/INTS6/hSSB1 complex in the DNA damage repair process.

Vidhyasagar et al. showed recently that the purified fulllength human INTS3 is a monomer in solution ${ }^{20}$. In contrast, our crystal structure of INTS3c/INTS6c complex indicated that the C-terminal region of INTS3 dimerizes and such a dimerization is independent of its interaction with INTS6c. Importantly, we showed that the two interface deletion mutants of INTS3 were observed to be diffused in the cytoplasm and failed to rescue the 


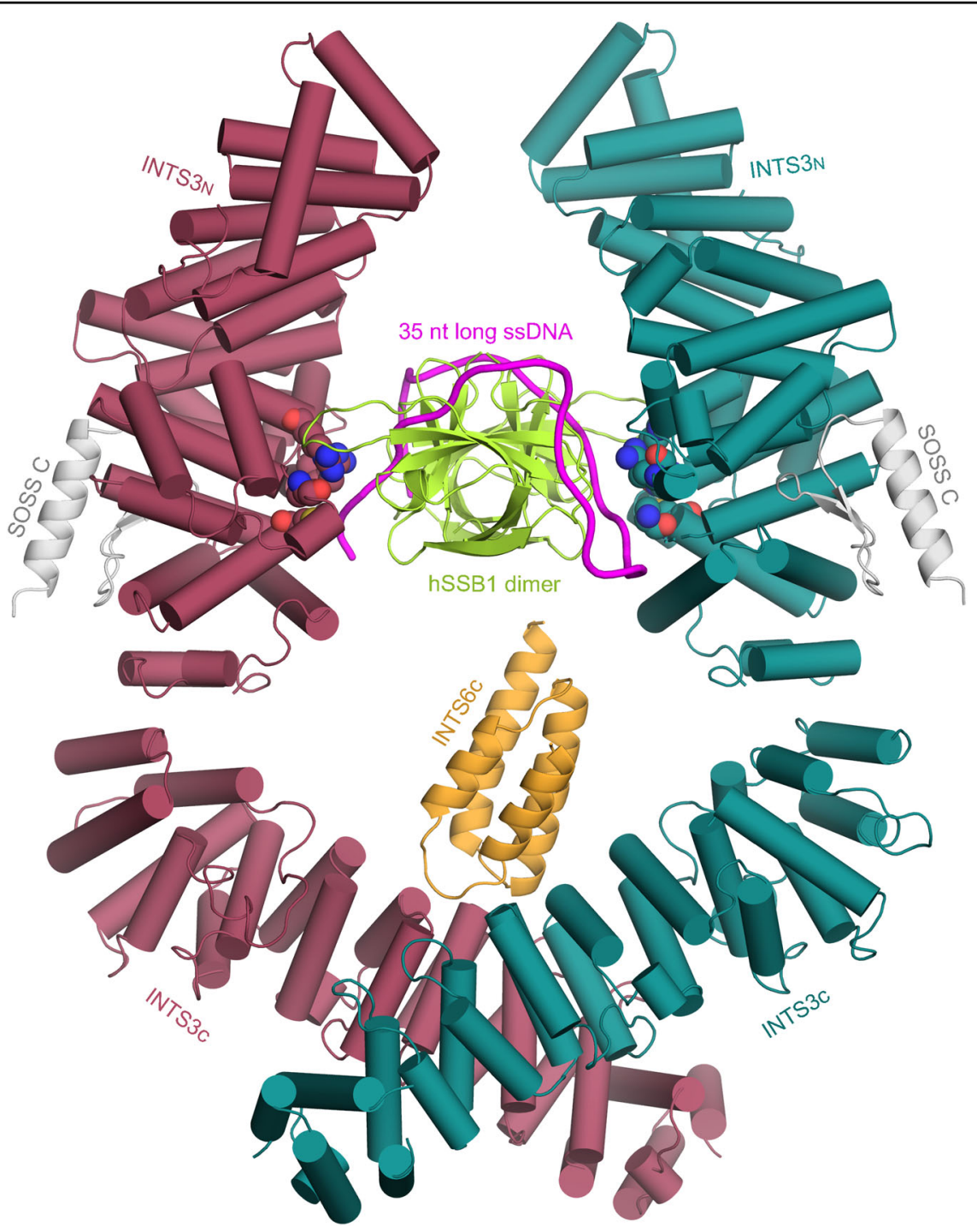

Fig. 6 The model of INTS3/hSSB1/SOSSC/INTS6c interacting with 35-nt long ssDNA. The color coding for INTS3 and INTS6c is the same as in Fig. 1. SOSSC and SSB1 are shown in light gray and lemon, respectively, while SsDNA is shown in magenta. The residues that were previously observed to contact SSDNA in the structure of INTS3 $_{N} / S S B 1 / S O S S C$ are shown in space-filling models.

formation of RAD51 foci, suggesting that INTS3 dimerization is critical for DSB repair.

The SOSS1 complex binds to ssDNA with a minimal length of $\sim 35$-nt and its DNA binding affinity is $\sim 30$-fold stronger than that of hSSB1 alone ${ }^{34}$. Structural studies of $\mathrm{INTS}_{\mathrm{N}} / \mathrm{hSSB} 1 / \mathrm{SOSSC}^{19}$ showed that hSSB1 solely contributes to the binding of ssDNA while neither SOSSC nor $\mathrm{INTS}_{\mathrm{N}}$ is critical for ssDNA binding, implying that the Cterminal half of INTS3 may be involved in the recognition of longer ssDNA. Consistent with this view, Vidhyasagar et al. showed that the C-terminus of INTS3 rather than INTS3 ${ }_{\mathrm{N}}$ binds to dT30 albeit with a weak affinity probably contributed by the positively charged residues at its extreme Cterminal region ${ }^{20}$. However, our SPR data showed that
INTS3(1-995)/hSSB1 and full-length INTS3/hSSB1 bind to dT48 (Supplementary Fig. S5) with comparable affinities, and purified INTS3c alone is unable to bind ssDNA (Supplementary Fig. S4d), suggesting that the C-terminus of INTS3 has no direct contribution to ssDNA binding. Instead, our EMSA and SPR results strongly indicated that the C-terminus of INTS3 is required for the enhanced binding of INTS3/hSSB1 to dT48 via dimerization. As depicted in Fig. 6, one simple explanation for the enhanced longer ssDNA binding is that the dimerization of INTS3 engages two hSSB1 molecules to form an hSSB1 dimer with a larger ssDNA recognition surface/channel for binding longer ssDNA. However, such an hSSB1 dimer is not compatible with the structure deposited in the PDB database 
(PDB IDs, 5D8E, and 5D8F; no accompanying paper has been published), which shows that hSSB1 dimerizes via its C-terminal tail. Furthermore, the superposition of INTS $3_{\mathrm{N}} /$ SSB1/SOSSC with hSSB1 (PDB codes: 5D8E and 5D8F) at SSB1 gives rise to severe steric clashes, suggesting that the hSSB1 dimer in the deposited structures might not be the physiologically relevant form.

Previously, it was reported that both $\mathrm{N}$ and $\mathrm{C}$ termini of INTS3 are important for its foci formation after the DNA damage, but its C-terminal deletion exhibited a stronger phenotype than its $\mathrm{N}$-terminal deletion ${ }^{17}$. Consistent with these results, our previous and present data showed that the N-terminus of INTS3 functions as a scaffold for SOSS1 assembly while the C-terminus of INTS3 dimerizes to promote the formation of a larger SOSS1 complex. The large and conserved INTS3 dimer interface plus its functional importance suggests that the dimer of INTS3 is likely to be its functional unit in DSB repair and is required for the assembly of a larger SOSS1 complex. Consistent with this notion, SOSS1 exists as more than one copy in vivo ${ }^{18}$. The larger SOSS1 complex formation mediated by INTS3 dimerization is not only required for foci formation but also important for recognizing longer ssDNA during the DSB repair process. Further structural and functional studies on the full-length INTS3 in complex with hSSB1 are required to clarify the mechanism governing the role of SOSS1 in DSB repair.

\section{Materials and methods}

Purification, crystallization, and structure determination of INTS3c/INTS6c

The C-terminal half of INTS3 consisting of residues 560995 was cloned into a pGEX-6p-1 vector, expressed in E. coli BL21 codonPlus cells, and purified to homogeneity using successive chromatographic steps involving GST affinity and gel filtration columns. However, the purified C-terminal of INTS3 was prone to degradation. In an effort to characterize the most stable fragment of INTS3, we used limited proteolysis followed by N-terminal sequencing to identify two stable fragments of INTS3, residues 560-905 and 915-995. These two fragments were co-expressed in pETDuet1 vector with GST tag attached to the first fragment (residues 560905) and the complex (INTS3c) was firstly purified by glutathione-Sepharose 4B followed by removal of the GST tag. INTS3C was further purified by Mono Q and Superdex-75 columns to $>95 \%$ purity. The INTS6 fragment consisting of residues 800-887 (INTS6c) was cloned into a pGEX-6p-1 vector, expressed in BL21 (DE3) codon Plus cells, and was purified using successive chromatographic steps involving GST affinity, GST tag cleavage by 3C Protease, and gel filtration columns. The purified INTS3c and INTS6c were mixed together and the resultant INTS3c/INTS6c complex was subjected to size-exclusion chromatography. The pooled fractions were concentrated to $15 \mathrm{mg} / \mathrm{ml}$ and subjected to crystallization screening. The INTS3c/INTS6c complex was crystallized in a buffer containing $1.7 \mathrm{M}$ ammonium citrate tribasic, $\mathrm{pH} 7.0$, and $4 \% \mathrm{v} / \mathrm{v}$ pentaerythritol ethoxylate $(3 / 4$ $\mathrm{EO} / \mathrm{OH})$. Before the data collection, the INTS3c/INTS6c crystals were cryo-protected by adding $25 \%$ glycerol to the crystallization buffer. As the sequences of INTS3c and INTS6c have no known homologs in the Protein Data Bank, we incorporated selenomethionine into INTS3c using feedback inhibition of methionine biosynthesis. The selenomethionine-incorporated crystals of INTS3c/INTS6c were crystallized in the same condition and diffracted to $2.6 \AA$ resolution. A diffraction dataset was collected at the Australian synchrotron, and the dataset was processed using $X D S^{35}$ and Aimless of $\mathrm{CCP} 4^{36}$. Selenomethionine sites were identified using HySS using a single anomalous dispersion method. The sites were refined using Resolve and phases were extended to the whole complex using PHASER in the PHENIX suite of crystallographic programs ${ }^{37}$. The resulting structure was used as a molecular replacement model for data collected on native INTS3c/INTS6c crystals that diffracted to $2.4 \AA$. The structure was further manually built in COOT and refined using phenix.refine to $R_{\text {work }}$ and $R_{\text {free }}$ values of 0.20 and 0.25 , respectively. The statistics of the structure determination and refinement are summarized in Supplementary Table S1.

\section{Cell culture and transfection}

HEK293T and HeLa cells were maintained in DMEM supplemented with $10 \%$ fetal bovine serum and $1 \%$ penicillin and streptomycin. Cell transfection was performed using Lipofectamine2000 (Invitrogen), following the manufacturer's protocol. The mammalian expression plasmids for SFB- or Myc-tagged INTS3 and INTS6 were previously described ${ }^{16}$. Site-directed mutagenesis was performed to obtain the INTS3, INTS6, or INTS3 mutants. For transient expression of INTS3, INTS6, or their mutants, the corresponding fragment in the entry vector was transferred into Gateway-compatible destination vector, which harbors either an N-terminal tripleepitope tag (S protein tag, Flag epitope tag, and Streptavidin binding peptide tag) or an $\mathrm{N}$-terminal Myc tag. All clones were sequenced to verify desired mutations.

\section{RNA interference}

All siRNAs were synthesized by Dharmacon Inc. The siRNAs were 21 base pairs and sequences are as follows: INTS3 siRNA: 5'-GAUGAGAGUUGCUAUGACAdTdT3', INTS6 siRNA: 5'-GGAAAGAAAUUGAUGCAUUdT dT-3', and control siRNA: 5' - UUCAAUAAAUUCUUGA GGUUU- $3^{\prime}$. The siRNA-resistant wild-type and mutant INTS3 constructs were generated by changing nine nucleotides in the INTS3 siRNA targeting region (G1569A, T1572C, G1575A, A1576T, G1577C, T1578A, C1581T, T1584C, and C1587T substitutions). The siRNA-resistant 
wild-type and mutant INTS6 constructs were generated by changing six nucleotides in the INTS6 siRNA targeting region (A2388T, G2391A, A2394G, G2397A, T2403C, and C2406G substitutions). The siRNA transfection was performed with $100 \mathrm{nM}$ siRNA duplexes using LipofectamineRNAiMAX following the manufacturer's instruction. Transfection was repeated twice with an interval of $24 \mathrm{~h}$ to achieve maximal RNAi effect.

\section{Antibodies}

Specific antibodies recognizing RAD51 were described previously $^{16}$. Anti-Myc (9E10) and anti-Flag (M2) antibodies were purchased from Covance and Sigma, respectively.

\section{Co-immunoprecipitation and western blotting}

For Flag immunoprecipitations, $0.8 \mathrm{ml}$ aliquot of lysate was incubated with $1 \mu \mathrm{g}$ of the Flag monoclonal antibody and $25 \mu \mathrm{l}$ of a 1:1 slurry of Protein A Sepharose for $2 \mathrm{~h}$ at $4{ }^{\circ} \mathrm{C}$. The Sepharose beads were washed three times with NTEN buffer, boiled in 2X SDS loading buffer, and resolved on SDS-PAGE. Membranes were blocked with $5 \%$ milk in TBST buffer and then probed with antibodies as indicated.

\section{Cell survival assays}

HeLa cells were transfected twice with control siRNA or siRNAs specifically targeting INTS3 or INTS6. Twentyfour hours after the second transfection, cells $\left(10^{3}\right)$ were split and transferred into $60 \mathrm{~mm}$ dishes. After incubation for $24 \mathrm{~h}$, cells were treated with IR as indicated. Twentyfour hours later, the medium was replaced and the cells were incubated for further $14 \mathrm{~d}$. The resulting cells were fixed and stained with Coomassie blue.

\section{Lentivirus packaging and infection}

Tet-On inducible SFB-tagged lentiviral vector and packaging plasmids (pMD2G and pSPAX2) were kindly provided by Prof. Songyang Zhou (Baylor College of Medicine). INTS3 entry constructs were transferred into the Gateway-compatible SFB-tagged lentiviral vector. Virus supernatant was collected $48 \mathrm{~h}$ after co-transfection of lentiviral vectors and packaging plasmids (pMD2G and pSPAX2) into HEK293T cells. Cells were infected with viral supernatants with the addition of $8 \mu \mathrm{g} \mathrm{ml}^{-1}$ polybrene (Sigma), and stable pools were selected with a medium containing $500 \mu \mathrm{g} \mathrm{ml}^{-1} \mathrm{G} 418$ (Calbiochem). The expression of the indicated genes in the stable pools was induced by the addition of $1 \mu \mathrm{g} \mathrm{ml}^{-1}$ doxycycline (Sigma) for $24 \mathrm{~h}$.

\section{Immunofluorescence staining}

Indirect immunofluorescence was carried out as described previously ${ }^{16}$. HeLa cells cultured on coverslips were treated with IR (10 Gy) for $6 \mathrm{~h}$. Cells were then washed with PBS, pre-extracted with buffer containing $0.5 \%$ Triton X-100 for $5 \mathrm{~min}$, and fixed with 3\% paraformaldehyde for $10 \mathrm{~min}$ at room temperature. Cells were incubated in primary antibody for $30 \mathrm{~min}$ at room temperature. After three times 5 min wash with PBS, a secondary antibody was added and incubated at room temperature for $30 \mathrm{~min}$. Cells were then stained with DAPI to visualize nuclear DNA. The coverslips were mounted onto glass slides with an anti-fade solution and visualized using a Nikon ECLIPSE i80 fluorescence microscope with a Nikon Plan Fluor 603 oil objective lens.

\section{Electrophoretic mobility shift assay}

INTS3(1-995)/hSSB1 was mixed with INTS6c at the molar ratio of $1: 1$ on ice for $1 \mathrm{~h}$. Then INTS3 $(1-500) /$ hSSB1, INTS3(full-length)/hSSB1, INTS3(1-995)/hSSB1/ INTS6c, INTS3(1-995)/hSSB1, or its mutants were incubated with 0.5 fmole of $6^{\prime}$-carboxy-fluorescein $\left(6^{\prime}\right.$ FAM) labeled dT48 at the molar ratio of 0.25:1, 0.5:1, 1:1, and 2:1 in the buffer $(50 \mathrm{mM} \mathrm{NaCl}, 20 \mathrm{mM}$ HEPES, $1 \mathrm{mM}$ $\mathrm{MgCl}_{2}, 0.5 \mathrm{mM}$ EDTA, and 0.2 mM DTT, pH 7.5) on ice for $1 \mathrm{~h}$. The reactions were resolved on $4-20 \%$ gradient native gel with $0.5 \mathrm{X}$ TBE buffer for $4 \mathrm{~h}$ at $110 \mathrm{~V}$. The gels were analyzed by Typhoon 8600 .

\section{Surface plasmon resonance (SPR)}

SPR measurements were performed at $25{ }^{\circ} \mathrm{C}$ using BIACORE T200 instrument and the data were analyzed using Graphpad Prism 7.0. INTS3(1-500)/hSSB1, INTS3 (1-995) /hSSB1, and INTS3(full length)/hSSB1 complex were immobilized on to the Series S NTA sensor chip in the running buffer (200 mM NaCl, $40 \mathrm{mM}$ HEPES, pH 7.5, $50 \mu \mathrm{M}$ EDTA). The manual injection process lasted for $30 \mathrm{~s}$ at $20 \mu \mathrm{min}^{-1}$ to reach an immobilization level of 1000RU. dT48 was dissolved in the running buffer in multiple proportion dilution. The contact time was $30 \mathrm{~s}$ for each concentration of dT48 and the disassociation time was $300 \mathrm{~s}$ at $30 \mu \mathrm{min}^{-1}$.

\section{Modeling the plausible interaction of SOSS1 complex with longer ssDNA}

Human SSB1 of INTS3 ${ }_{\mathrm{N}} / \mathrm{hSSB} 1 / \mathrm{SOSSC} /$ ssDNA (PDB ID: $4 \mathrm{OWW}$ ) was superposed on subunits I and II of $P$. falciparum SSB (PDB ID: 3ULP) to generate a dimeric hSSB1/INTS3 ${ }_{\mathrm{N}}$ complex. Furthermore, the two ssDNA fragments bound to subunits I and II of Pf SSB were manually connected by building intervening nucleotides such that $\sim 35$-nt long ssDNA is wound around the dimeric hSSB1. The resulting dimeric hSSB1/INTS3 ${ }_{\mathrm{N}} /$ ssDNA complex was subjected to unrestrained molecular dynamics simulations to optimize the interactions between hSSB1 and ssDNA. The final model of the SOSS1 complex interacting with a 35-nt ssDNA was generated by aligning the dyad axis of dimeric $\mathrm{hSSB} 1 / \mathrm{INTS} 3_{\mathrm{N}}$ with that 
of INTS3c/INTS6c complex such that the C-terminus of INTS $3_{\mathrm{N}}$ is close to the $\mathrm{N}$-terminus of the INTS3c with appropriate contacts.

\section{Acknowledgements}

We would like to thank the beamline scientists at MX2, Australian Synchrotron for assistance and access to synchrotron radiation facilities. We thank Dr. Roland Gamsajaeger at Western Sydney University for sharing the tetrameric model of hSSB1. This work was supported by Natural Science Foundation of China (31470723 to H.S.) and the Agency for Science, Technology, and Research in Singapore (H.S.).

\section{Author details}

MOE Laboratory of Biosystems Homeostasis and Protection and Innovation Center for Cell Signaling Network, Life Sciences Institute, Zhejiang University, Hangzhou, Zhejiang, China. ${ }^{2}$ Institute of Molecular and Cell Biology, 61 Biopolis Drive, Singapore City, Singapore. ${ }^{3}$ Department of Biochemistry, National University of Singapore, 14 Science Drive, Singapore City, Singapore

\section{Author contributions}

H.S. and J.H. conceived and coordinated the study. Y.J., Z.C., S.R.B., Q.S. and N.S. performed the experiments. Y.J., Z.C., S.R.B., J.H. and H.S. analyzed the data and wrote the paper.

\section{Data availability}

The atomic coordinates of INTS3c/INTS6c and the corresponding structure factors are deposited at the PDB with the accession code, 7BV7.

\section{Conflict of interest}

The authors declare no competing interests.

\section{Publisher's note}

Springer Nature remains neutral with regard to jurisdictional claims in published maps and institutional affiliations.

Supplementary information The online version contains supplementary material available at https://doi.org/10.1038/s41421-021-00283-0.

Received: 13 December 2020 Accepted: 17 May 2021

Published online: 17 August 2021

\section{References}

1. Aguilera, A. \& Gomez-Gonzalez, B. Genome instability: a mechanistic view of its causes and consequences. Nat. Rev. Genet. 9, 204-217 (2008).

2. van Gent, D. C., Hoeijmakers, J. H. \& Kanaar, R. Chromosomal stability and the DNA double-stranded break connection. Nat. Rev. Genet. 2, 196-206 (2001).

3. Jackson, S. P. \& Bartek, J. The DNA-damage response in human biology and disease. Nature 461, 1071-1078 (2009).

4. McKinnon, P. J. DNA repair deficiency and neurological disease. Nat. Rev. Neurosci. 10, 100-112 (2009).

5. Symington, L. S. \& Gautier, J. Double-strand break end resection and repair pathway choice. Annu. Rev. Genet. 45, 247-271 (2011).

6. Ceccaldi, R., Rondinelli, B. \& D'Andrea, A. D. Repair pathway choices and consequences at the double-strand break. Trends Cell Biol. 26, 52-64 (2016).

7. Chapman, J. R., Taylor, M. R. \& Boulton, S. J. Playing the end game: DNA double-strand break repair pathway choice. Mol. Cell 47, 497-510 (2012).

8. Huertas, P. DNA resection in eukaryotes: deciding how to fix the break. Nat. Struct. Mol. Biol. 17, 11-16 (2010).

9. Krejci, L., Altmannova, V., Spirek, M. \& Zhao, X. Homologous recombination and its regulation. Nucleic Acids Res. 40, 5795-5818 (2012).

10. Jazayeri, A., Balestrini, A., Garner, E., Haber, J. E. \& Costanzo, V. Mre11-Rad50Nbs1-dependent processing of DNA breaks generates oligonucleotides that stimulate ATM activity. EMBO J. 27, 1953-1962 (2008).
11. Lee, J. H. \& Paull, T. T. ATM activation by DNA double-strand breaks through the Mre11-Rad50-Nbs1 complex. Science 308, 551-554 (2005).

12. Zou, Y., Liu, Y., Wu, X. \& Shell, S. M. Functions of human replication protein A (RPA): from DNA replication to DNA damage and stress responses. J. Cell Physiol. 208, 267-273 (2006).

13. Richard, D. J. et al. Single-stranded DNA-binding protein hSSB1 is critical for genomic stability. Nature 453, 677-681 (2008).

14. Richard, D. J. et al. hSSB1 interacts directly with the MRN complex stimulating its recruitment to DNA double-strand breaks and its endo-nuclease activity. Nucleic Acids Res. 39, 3643-3651 (2011).

15. Richard, D. J. et al. hSSB1 rapidly binds at the sites of DNA double-strand breaks and is required for the efficient recruitment of the MRN complex. Nucleic Acids Res. 39, 1692-1702 (2011).

16. Huang, J., Gong, Z., Ghosal, G. \& Chen, J. SOSS complexes participate in the maintenance of genomic stability. Mol. Cell 35, 384-393 (2009).

17. Zhang, F., Wu, J. \& Yu, X. Integrator3, a partner of single-stranded DNA-binding protein 1, participates in the DNA damage response. J. Biol. Chem. 284, 30408-30415 (2009)

18. Li, Y. et al. HSSB1 and hSSB2 form similar multiprotein complexes that participate in DNA damage response. J. Biol. Chem. 284, 23525-23531 (2009).

19. Ren, W. et al. Structural basis of SOSS1 complex assembly and recognition of ssDNA. Cell Rep. 6, 982-991 (2016).

20. Vidhyasagar, V. et al. Biochemical characterization of INTS3 and C9ORF80, two subunits of hNABP1/2 heterotrimeric complex in nucleic acid binding. Biochem. J. 475, 45-60 (2018).

21. Zhang, F., Ma, T. \& Yu, X. A core hSSB1-INTS complex participates in the DNA damage response. J. Cell Sci. 126, 4850-4855 (2013).

22. Krissinel, E. \& Henrick, K. Inference of macromolecular assemblies from crystalline state. J. Mol. Biol. 372, 774-797 (2007).

23. Richard, D. J., Bolderson, E. \& Khanna, K. K. Multiple human single-stranded DNA binding proteins function in genome maintenance: structural, biochemical and functional analysis. Crit. Rev. Biochem. Mol. Biol. 44, 98-116 (2009).

24. Paquet, $\mathrm{N}$. et al. hSSB1 (NABP2/OBFC2B) is regulated by oxidative stress. Sci. Rep. 6, 27446 (2016).

25. Touma, C. et al. A data-driven structural model of hSSB1 (NABP2/OBFC2B) selfoligomerization. Nucleic Acids Res. 45, 8609-8620 (2017).

26. Raghunathan, S., Kozlov, A. G., Lohman, T. M. \& Waksman, G. Structure of the DNA binding domain of E. coli SSB bound to ssDNA. Nat. Struct. Biol. 7, 648-652 (2000).

27. Antony, E., Weiland, E. A., Korolev, S. \& Lohman, T. M. Plasmodium falciparum SSB tetramer wraps single-stranded DNA with similar topology but opposite polarity to E. coli SSB. J. Mol. Biol. 420, 269-283 (2012).

28. George, N. P. et al. Structure and cellular dynamics of Deinococcus radiodurans single-stranded DNA (ssDNA)-binding protein (SSB)-DNA complexes. J. Biol. Chem. 287, 22123-22132 (2012).

29. Baillat, D. et al. Integrator, a multiprotein mediator of small nuclear RNA processing, associates with the C-terminal repeat of RNA polymerase II. Cell 123, 265-276 (2005).

30. Huertas, P. \& Aguilera, A. Cotranscriptionally formed DNA:RNA hybrids mediate transcription elongation impairment and transcription-associated recombination. Mol. Cell 12, 711-721 (2003)

31. Ohle, $C$. et al. Transient RNA-DNA hybrids are required for efficient doublestrand break repair. Cell 167, 1001-1013 (2016).

32. Springer, T. A. Complement and the multifaceted functions of WWA and integrin I domains. Structure 14, 1611-1616 (2006).

33. Cotta-Ramusino, $C$. et al. A DNA damage response screen identifies RHINO, a 9-1-1 and TopBP1 interacting protein required for ATR signaling. Science 332, 1313-1317 (2011)

34. Yang, S. H. et al. The SOSS1 single-stranded DNA binding complex promotes DNA end resection in concert with Exo1. EMBO J. 32, 126-139 (2013).

35. Kabsch, W. XDS. Acta Crystallogr. D 66, 125-132 (2010).

36. Winn, M. D. et al. Overview of the CCP4 suite and current developments. Acta Crystallogr. D 67, 235-242 (2011).

37. Liebschner, D. et al. Macromolecular structure determination using X-rays, neutrons and electrons: recent developments in Phenix. Acta Crystallogr. D 75 861-877 (2019). 